\title{
GPS Technology and Human Psychological Research: A Methodological Proposal
}

\author{
Pedro S. A. Wolf and W. J ake J acobs \\ University of Arizona
}

Animal behaviorists have made extensive use of GPS technology since 1991. In contrast, psychological research has made little use of the technology, even though the technology is relatively inexpensive, familiar, and widespread. Hence, its potential for pure and applied psychological research remains untapped. We describe three methods psychologists could apply to individual differences research, clinical research, or spatial use research. In the context of individual differences research, GPS technology permits us to test hypotheses predicting specific relations among patterns of spatial use and individual differences variables. In a clinical context, GPS technology provides outcome measures that may relate to the outcome of interventions designed to treat psychological disorders that, for example, may leave a person homebound (e.g. Agoraphobia, PTSD, TBI). Finally, GPS technology provides natural measures of spatial use. We, for example, used GPS technology to quantify traffic flow and exhibit use at the Arizona Sonora Desert Museum. Interested parties could easily extend this methodology some aspects of urban planning or business usage.

Keywords: GPS, Ethology, Spatial Cognition, Outcome Evaluation, Human Navigation

The United States government initially developed Global Positioning System (GPS) technology as a military navigational tool. In 1983, Soviet aircraft shot down a civilian airliner after it strayed into restricted airspace due to navigational errors. Thereafter, the US government authorized civilian use of GPS technology (Shroeer \& Elena 2000). Although not fully functional for military use until the mid 1990's, when a complete 24-satellite constellation was in orbit, a degraded signal was available for civilian use. In May of 2000, both military and civilians receive access to the fully functional (with some exceptions) military-quality signals.

Since then, GPS technology has penetrated the civilian world. We find GPS technology in our cellular phones, our 911 emergency systems, our cars, as a work tool in surveying, mining, shipping, truck driving, and other jobs that require navigational aids or need recordings of geographic locations. GPS technology has also penetrated scientific research. we find GPS technology commonly used in the Geosciences (e.g., studying plate tectonics), Climatology (e.g., measuring glacial ice flow), and Biology (e.g., tracking animals).

Unfortunately, most Psychologists overlook this technology. To date, published articles using GPS technology in human psychology investigate the viability of using specific equipment in urban settings (e.g. GPS enabled cellular phones)(Michaels, McGregor, Allen, \& Fickas, 2008) or study populations and their movement using data from cellular phone networks (Gonzalez, Hidalgo, \& Barabasi, 2008), but little else. To rectify this, we outline examples of how psychologists might use GPS technology in Personality Research: (individual differences), Psychopathology (clinical outcomes), or Environmental Psychology (spatial use).

\section{Psychological Methods}

Behavioral methods lie at the core of both 'pure' (e.g., experimental) and 'applied (e.g., clinical) psychology. The hope is that pure and applied psychology will inform each other.

Unfortunately, seemingly unbridgeable methodological gaps exist between these fields. Pure psychology relies primarily on experimental designs whereas applied relies primarily on 
naturalistic, correlational, and quasi-experimental designs. A few pure psychologists criticize applied psychology for overreliance on non-experimental methods - suggesting that the latter cannot develop adequate etiological models of psychopathology. Conversely, a few applied psychologists criticize pure psychology for an overreliance on laboratory-based work suggesting that results from the laboratory may not generalize to important areas of every-day life. Unfortunately, these assertions remain largely abstract. Although solid observational methodologies exist (e.g., Jacobs, et al. 1988), the meager amount of work examining the generalizability of theoretical work coming from the cognitive lab, or the veracity of theory coming from clinical psychology, anchors itself in correlations among various forms of selfreport. Bluntly put, observational methodologies are difficult to use, labor intensive, expensive, and, all too often, reactive.

Self-report methodologies can be psychometrically sound (e.g., Hopko, Lejuez, Ruggiero, \& Eifert, 2003), making them useful in pure and applied investigations of anxiety, pain, alcohol abuse, sexual behavior, gambling, insomnia, and sickle-cell disease (e.g., Nelson \& Clum 2002; van den Brink, Bandell, \& Huijer, 2001; Watson, 1999; Okami, 2002; Atlas \& Peterson, 1990; Haythornwaite, Hegel, \& Kerns, 1991; Ely, Dampier, Gilday, O’Neal, \& Brodecki, 2001). At the same time, self-report methods can be subject to memory distortions, influenced by social desirability in all of its forms, and, like direct observational methods, highly reactive (e.g., Patterson \& Sechrest, 1983; Cone, 1978). Nevertheless, for those interested in what people do in their natural environment, few practical alternatives to self-report existed.

Recent improvements in Global Positioning Satellite (GPS) and cell phone technology occurred (e.g., Goodwin, Velicer, \& Intille, 2008) changed all of that. Although still in its infancy, the use of GPS technology, perhaps married with others (e.g., an Electronically Activated Recorder (EAR) a technology that samples ambient sounds in the participant's daily life), increases the researcher ability to gather some forms of real-time naturalistic data unobtrusively and without the memory distortions, demand characteristics, and reactivity often introduced by self-report (Mehl, Pennebaker, Crow, Dabbs, \& Price, 2001).

\section{Individual Differences Methods}

A main strength of GPS technology is that it easily records day-to-day and/ or second-bysecond spatial activity. When theory predicts specific behavior patterns, one may use these records as an outcome or predictor variable in correlational or quasi-experimental research.

Most commercially available GPS receivers record the date, time, latitude, longitude, speed, and altitude at given time intervals. These intervals can vary from second-by-second to every $\mathrm{x}$ number of days depending on the device and its capability. The interval a researcher records depends entirely on the research question. For example, if the researcher tracks the migratory behavior of elephants, a daily or weekly recording of the elephants' position will suffice and is preferable to more frequent measurement, because longer intervals help conserve battery life. If the researcher tracks daily activities that include five-minute trips to the gas station, a more frequent time interval is preferred.

In addition to choosing appropriate time intervals, the researcher must decide how to transform a mass of raw data into something theoretically meaningful. Transforming the discrete data points given by a GPS unit into continuous variables is necessary because most naturalistic research involves correlational methods of one sort or another. For our research, it is necessary to use a large (up to about 300,000 observations per individual) data set to estimate the size and shape of each individual's home range, to determine the boundaries of an individual's movement during the course of everyday activities, and to determine the number of places the individual visits. 


\section{GPS AND PSYCHOLOGICAL RESEARCH}

\section{Measuring Distance Traveled}

Another measure of spatial use is the distance a person covers per unit of time. All that is necessary to obtain this measure is to record the distance between one point and another, add that distance to the distance between point $\mathrm{A}$ and point $\mathrm{B}$, continue doing this until all the distances are measured and added, and divide the sum by the desired unit of time. This feature is built into many of the programs that come with commercially available GPS devices.

\section{Minimum Convex Polygon Method}

We use a minimum convex polygon (MCP) method to estimate a rough and computationally efficient measure of an individual's home range. An MCP is the minimum number of points necessary to encompass all other points plotted on a plane. After obtaining all of the points that will make up an MCP, the researcher can calculate the area of the minimum convex polygon which serves as a measure of that individual's home range.

This method is computationally efficient because the program used to estimate home ranges needs only to identify the data points and measure one polygon. Other methods are more complex, depending on the size of the data set, computationally inefficient, and take considerable time to calculate, even when using high-performance (super) computers.

We use a statistical package available in the R software environment to instantiate the MCP estimation method freely available from http://cran.r-project.org/ . Down-loading the program provides an immediately functional statistical tool. To perform analyses that are not part of the R-data system, the data analyst downloads specific packages. For example, we used the "adehabitat" package from http:// cran. r-project.org/ web/ packages/adehabitat/index.html to calculate the MCP for each individual's home range (see Figure 1).

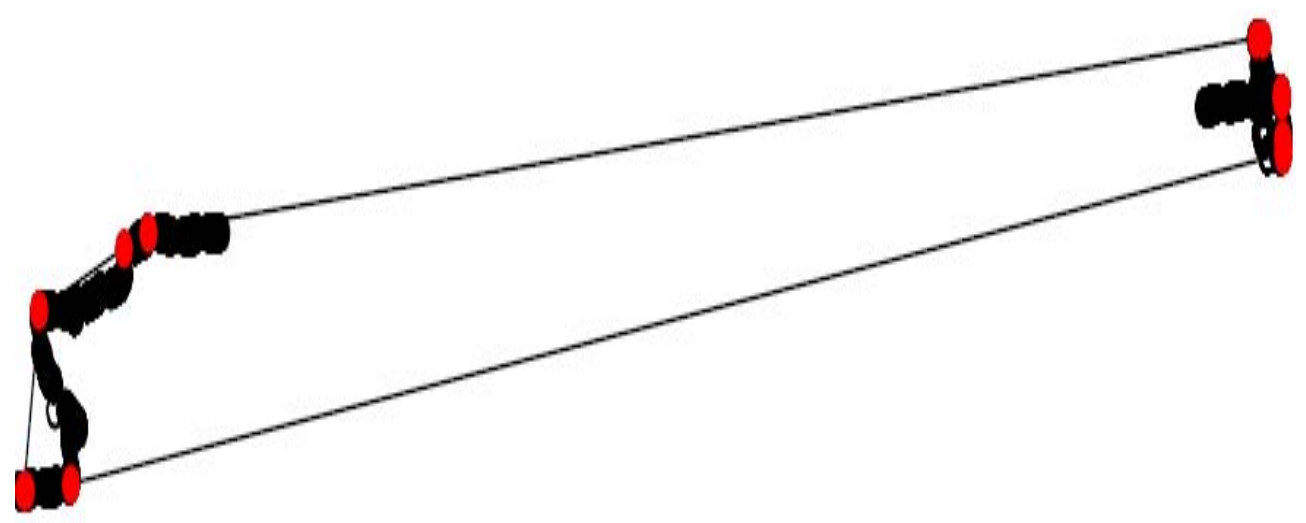

Figure 1. Minimum convex polygon. The red points are the minimum number of points necessary to encompass the rest of the points in this individual's data set.

Notice, in Figure 1, there is a sizable area in the middle of the plot that the individual does not visit. Unfortunately, the MCP method includes this empty area as part of the individual's home range. Although computationally efficient, the MCP method is not an appropriate measure of an individual's home range because the home range can be split into two areas 
separated by an area used only for travel. Hence, the MCP method can grossly overestimate area of the home range.

\section{Nearest Neighbor Convex Hull (NNCH)}

The MCP method can overestimate home range (see Figure 1). To address the problem, we use a variant of the MCP method included in the "adehabitat" package. The Nearest Neighbor Convex Hull (NNCH) method enables researchers to obtain a more precise estimate of the area(s) the participant actually visits. The method does this by creating a k-NNCH covering. This covering provides a more precise representation of an individual's home range. A k-NNCH covering is constructed out of the dataset's points by creating individual polygons composed of each point and its nearest $(\mathrm{k}-1)$ neighbors and then uniting all of these individual polygons. The area of the k-NNCH is then calculated.

The analyst may increase or decrease the sensitivity to empty space by decreasing or increasing the value for $\mathrm{k}$. In essence, the closer $\mathrm{k}$ is to the number of points in the data set, the less sensitive to empty space the estimate becomes. One way to think of this sensitivity to empty space is that as $\mathrm{k}$ increases the value becomes closer to the number of points necessary to define the entire dataset's MCP (see Getz \& Wilmers 2004).

Although controlling the sensitivity to "unused" space in a home range estimation is useful, there is a practical limitation of this method. As stated previously, the data sets obtained by GPS units can be large. The iterative nature of the $\mathrm{NNCH}$ method makes it computationally inefficient; the amount of computational power necessary to estimate these home ranges increases as an exponential function of the number of data points per individual data set. Even with access to high-performance computers, it can take hours to obtain an estimate of an individual's home range. An alternative approach is to sample from the large data set. In our experience a sample of 500 to 600 points is the optimum sample size which balances computation time with accuracy. Figure 2 is an example of a plot using the NNCH method in combination with the sampling procedure.

\section{Measuring Number of Places Visited}

GPS technology makes it possible to record the number of places a person visited over a given time. One way to do this is to plot a $\mathrm{NNCH}$ with a small $\mathrm{k}$ parameter and count the number of high-density areas on the plot. Another is to use the speed variable and find the number of places the person did not move during a predefined time. A program that came with our LandSeaAir Systems GPS devices produced a list of places (geographic, businesses, residence, and the like) the person visited - including the address, time spent at place, and distance between places.

\section{Data Aggregation}

Each method used to aggregate these data comes with its own biases. We typically chose a multivariate approach to address this problem. Instead of calculating bivariate correlations between individual predictor and outcome variables, we estimated a predictor and an outcome by aggregating measures of each. We do so by calculating unit weighted factor scores for the predictor and outcome measures. After calculating these multivariate estimates of the constructs, we correlated them. This provides a convenient and powerful method for estimating relations among such variables. 


\section{GPS AND PSYCHOLOGICAL RESEARCH}

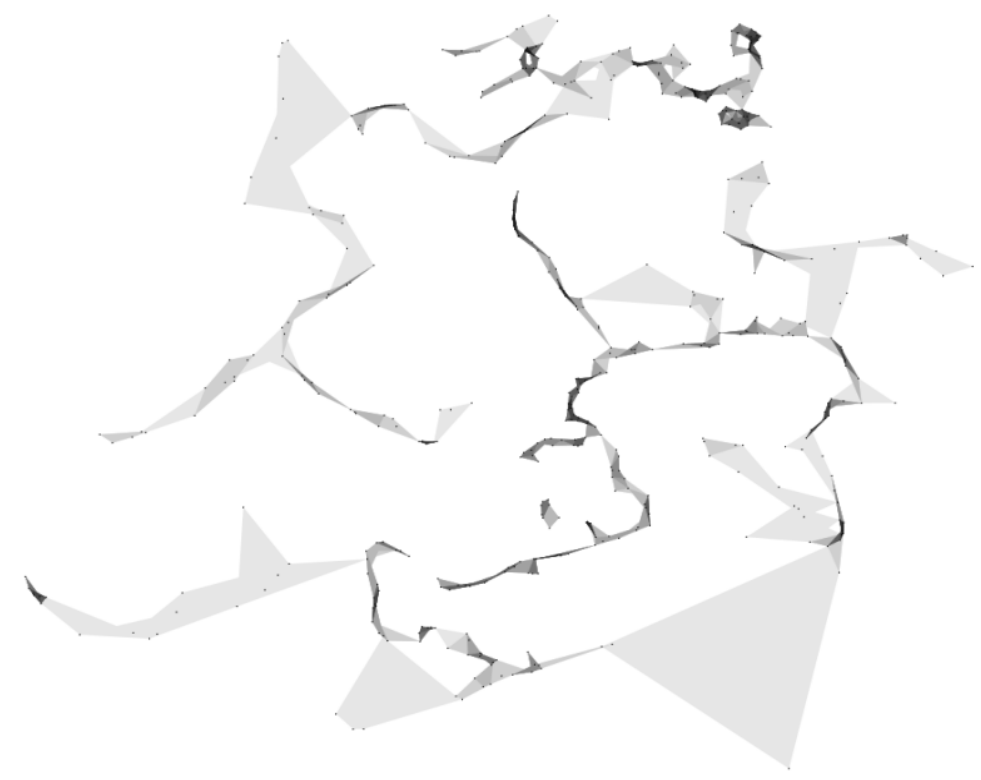

Figure 2. Nearest Neighbor Convex Hull plot. where $\mathrm{k}=7$ on a 500 point dataset. The individual was walking in an outdoor zoo environment. The darker areas represent places with higher densities of points. A simple MCP method would include the entire zoo rather than the paths taken by the individual.

\section{Future Directions}

Clinical Outcome Measure. We intend to use this approach to measure relations between disorders such as major depression, agoraphobia, PTSD, and spatial use - as well as one measure in a battery design to measure the outcome of interventions designed to help individual's who suffer from psychological disorders predicted to leave individuals homebound. For example, one could give a GPS unit to individuals suffering from Agoraphobia or PostTraumatic Stress Disorder, record base-rate of spatial use, provide an intervention, and examine the impact of the intervention on the individual's use of space - the size of the home range, the number of places visited, the kinds of places visited, the time spent away from home, and the like. If the treatment has an effect, then the way in which the individual uses space should change - for the better.

Spatial Use Research. We have used GPS technology in a more applied setting, primarily as Environmental Psychologists. In partnership with the Arizona Sonora Desert Museum, located in Tucson, Arizona, we recorded traffic flow in the museum. Although the analysis is not yet complete, it is obvious that visitors overuse some parts of the museum and underuse others. In collaboration with the Museum Directors, we are designing interventions to change the patterns of visitor flow and use. Importantly, we intend to track the impact of each intervention using counterbalanced sequence ABBA designs. As we advance our data analytic methods, we further incorporate some of the more sophisticated home range estimation techniques. By overlaying the density plots provided by those estimate techniques and an accurate museum 
WOLF AND J ACOBS

map, we, and our collaborative administrators, can see paths and exhibits that receive traffic and those that do not.

Technological Improvements. GPS technology alone offers only a small window on daily human behaviors. All a GPS unit can tell us is where a person goes, how s/ he got there, how long s/he stays, and when s/he leaves. The units do not tell us what these individuals are doing. To address that shortcoming, we recently developed technology that integrates GPS recordings and the ambient sound sampling method developed by Mehl et al. (2003, 2006, 2007). Mehl and his colleagues have used their ambient sound sampling technology, in which participants wear a recording device that samples their conversations throughout the day, to great effect (e.g., Mehl et al., 2003, 2006, 2007; Vazire \& Mehl, 2008). We hope a melding of GPS and sound recording technologies will further improve our ability to assess naturalistic behavior easily, inexpensively, and unobtrusively.

\section{References}

Atlas, G. D., \& Peterson, C. (1990). Explanatory style and gambling: how pessimists respond to losing wagers. Behaviour Research and Therapy, 28, 523- 529.

Coyne, J. C. (1976). Toward an interactional description of depression. Psychiatry: Interpersonal and Biological Processes, 39, 28- 40.

Ekers, D., Richards, D., \& Gilbody, S. (2008). A meta-analysis of randomized trials of behavioral treatment of depression. Psychological Medicine, 38, 611- 623.

Ely, B., Dampier, C., Gilday, M., O’Neal, P., \& Brodecki, D. (2002). Caregiver report of pain in infants and toddlers with sickle cell disease: reliability and validity of a daily diary. J ournal of Pain, 3, 50- 57.

Ferster, C. B. (1973). A functional analysis of depression. American Psychologist 28, 857- 870.

Getz, W. M., \& Wilmers, C. C. (2004). A local nearest-neighbor convex-hull construction of home ranges and utilization distributions. Ecography, 27, 489-505.

Gonzalez, M., Hidalgo, C., \& Barabasi, A. (2008). Understanding individual human mobility patterns. Nature, 453, 779-782.

Goodwin, M., W. Velicer, \& S. Intille. (2008). Telemetric monitoring in the behavior sciences. Behavior Research Methods, 40, 328-341.

Haythornthwaite, J . A., Hegel, M. T., \& Kerns, R. D. (1991). Development of a sleep diary for chronic pain patients. J ournal of Pain and Symptom Management, 6, 65- 72.

Hopco, D. R., \& Mullane, H. C. (2008). Exploring the relation of depression and overt behavior with daily diaries. Behaviour Research and Therapy, 46, 1085- 1089.

Hopko, D. R., Lejuez, C. W., Ruggiero, K. J., \& Eifert, G. H. (2003). Contemporary behavioral activation treatments for depression: procedures, principles, progress. Clinical Psychology Review, 23, 699- 717.

J acobs, W. J., Blackburn, J. R., Buttrick, M., Harpur, T. J., Kennedy, D., Mana, M. J., MacDonald, M. A., McPherson, L. M., Paul, D., \& Pfaus, J . G. (1988). Observations. Psychobiology, 16, 3-19.

Joiner, T. E., Jr. (1997). Shyness and low social support as interactive diatheses, with loneliness as mediator: testing an interpersonal-personality view of vulnerability to depressive symptoms. J ournal of Abnormal Psychology 106, 386- 394.

Lewinsohn, P. M. (1974). A behavioral approach to depression. In R. M. Friedman, \& M. M. Katz Eds., The Psychology of Depression: Contemporary Theory and Research. New York: Wiley.

Lewinsohn, P. M., \& Graf, M. (1973). Pleasant activities and depression. Journal of Consulting and Clinical Psychology, 41, 261- 268.

Martell, C. R., Addis, M. E., \&J acobson, N. S. (2001). Depression in context: Strategies for guided action. New York: W.W. Norton.

Mehl, M., \& Pennebaker, J. (2003). The social dynamics of a cultural upheaval: Social interactions surrounding September 11, 2001. Psychological Science, 146, 579-585.

Mehl, M., S. Gosling, \& Pennebaker, J. (2006). Personality in its natural habitat: Manifestations and implicit folk theories of personality in daily life. Journal of Personality and Social Psychology, 90, 862-877. 


\section{GPS AND PSYCHOLOGICAL RESEARCH}

Mehl, M., Pennebaker, J., Crow, D., Dabbs, J ., \& Price, J . (2001). The Electronically Activated Recorded (EAR): A device for sampling naturalistic daily activities and conversations. Behavior Research Methods, Instruments and Computers, 33, 517-523.

Mehl, M., Vazire, S., Ramírez-Esparza, N., Slatcher, R., \& Pennebaker, J . (2007). Are women really more talkative than men? Science, 317 (5834), 82.

Michaels, Y., McGregor, E., Allen, J., \& Fickas, S. (2008). Observing outdoor activity using global positioning system-enabled cell phones. Lecture Notes in Computer Science, 5120, 177-184.

Nelson, W. A., \& Clum, G. A. (2002). Assessment of panic frequency: reliability and validity of a time-line follow-back method. J ournal of Psychopathology and Behavioral Assessment, 24, 47- 54.

Okami, P. (2002). Dear diary: a useful but imperfect method. In M.W. Wiederman, \& B. E. Whitley, Jr. Eds., Handbook for Conducting Research on Human Sexuality pp. 195- 207. Mahwah, NJ : Lawrence Erlbaum.

Patterson, D., \& Sechrest, L., (1983). Nonreactive measures in psychotherapy outcome research. Clinical Psychology Review, 3, 391-416.

Shroeer D. \&Elena, M. (2000). Technology Transfer. Aldershot, England: Ashgate Publishing.

van den Brink, M., Bandell, H., \& Huijer, A. H. (2001). The occurrence of recall bias in pediatric headache: a comparison of questionnaire and diary data. Headache, 1, 11- 20.

Vazire, S., \& Mehl, M. (2008). Knowing me, knowing you: The accuracy and unique predictive validity of self-ratings and other-ratings of daily behavior. Journal of Personality and Social Psychology, 95, 1202-1216.

Watson, H. E. (1999). Problem drinkers in acute care settings: validation of an assessment instrument. International J ournal of Nursing Studies, 36, 415- 423. 